\title{
Multicentral meningothelial meningioma: A case of death
}

\author{
Mansueto Gelsomina $^{*}$, Emanuele Capasso ${ }^{2}$, Antonio Lombardi ${ }^{3}$, Pagliuca Francesca ${ }^{1}$, Pieri Maria ${ }^{2}$ and Claudio Buccelli $^{2}$ \\ ${ }^{1}$ Department of Advanced Biomedical Sciences, Pathology Section, University of Naples Federico II, Italy \\ ${ }^{2}$ Legal Medicine Section, University of Naples Federico II, Italy \\ ${ }^{3}$ Legal adviser of the Court of Naples, Italy
}

\begin{abstract}
Meningiomas are intracranial tumors originating from arachnoidal cells. Multiple intracranial meningiomas are rare and occur when two or more lesions occur either simultaneously or sequentially in different locations. We described a case of multiple meningothelial meningioma post forensic autopsy.
\end{abstract}

\section{Introduction}

Meningiomas are intracranial tumors originating from arachnoidal cells. Multiple intracranial meningiomas are rare and occur when two or more lesions are found either simultaneously or sequentially in different locations [1]. We describe a case of multiple meningothelial meningioma post forensic autopsy.

\section{Case report}

We have described a case of a 43-year-old male found dead in his bedroom by his parents and apparently decessed for unknown causes. Near the body hand-written suicide notes reporting his last wills and testament were found. Although the decedent lived together with his parents, he conducted a completely isolated life. Despite this, no clinical symptoms were documented, but only diagnostic hypothesis of depressive syndrome and double personality disorder (unmotivated aggressiveness and anger) referred by his parent. Nothing in the patient's history was suggestive for the presence of tumor. The autopsy was performed two days after death. The macroscopic appearance showed occipital hematoma and hemorrhagic punctuation pigmented surface of the left occipital the left parietal lobes, and temporal lobe. Tortuosity of the superficial vascular structures of the occipital lobes and small papillary vegetations millimetric in size on the right frontal lobe in the parasagittal were present, in correspondence of which, the cut section of the cortical area showed brownish with loss of substance (Figure 1-4). The sub-meningeal vessels showed clots. Sampling of brain areas showed in the two hemispheres an overall normal corticomedullary structure and multiple diffuse areas characterized by marked edema and fibrosis of the arachnoid layer of the meninges with dilated and congested vessels, images of angiomatosis and cortical edema (Figure 5). Other areas, particularly those described macroscopically as papillary proliferations, appeared as micropapillary structures with angiomatous stroma and with hyaline aspects (Figure 6), covered with epithelium that consisted in meningoendothelial cells with occasional psammoma-like bodies (Figure 6). These epitelioid elements had a syncytial-like organization (Figure 7). The micropapillae with edematous and fibrous stroma and in the angiomatosis areas are lined by cellular elements characterized by finely distributed nuclear chromatin. In some cases conspicuous nucleoli with clear vacuolation and secretory vacuoles in the cytoplasm were found (Figure 7); some pigment was observed. Immunohistochemistry showed a diffuse positivity for anti-vimentin (VIM) and focal for anti-EMA (Figure 8). These morphological features are consistent with a neoplasm with its origin in the arachnoid cells of the meninges, and configure the framework of "meningothelial variant" of meningiona in the multicentric form considering the spread and $\mathrm{j}$ 'ump localization of the lesions.

The other organs showed a conserved structure; the pulmonary sections, in particular, showed emphysematous aspects and diffuse
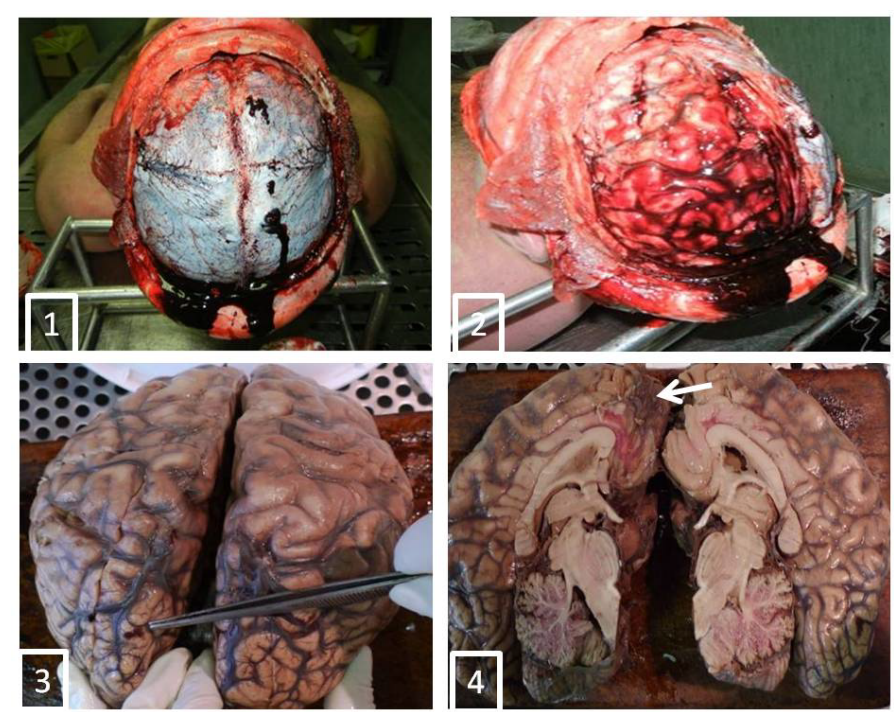

Figure 1-4. Aemorrage and tortuosity of the superficial vascular structures of the occipital lobes (Figures 1,2) and small vegetations papillary millimetric sizes on the right frontal lobe in the parasagittal were present (Figures 2,3), in correspondence of which, the cut section of the cortical area showed brownish with loss of substance (Figure 4, arrow).

Correspondence to: Mansueto Gelsomina, Department of Advanced Biomedical Sciences, University Federico II of Naples, Via Pansini 5, 80131 Naples, Italy, Tel: +390817463431, Fax: +390817463475, E-mail: mansueto.g@inwind.it

Received: July 14, 2016; Accepted: September 07, 2016; Published: September 12,2016 

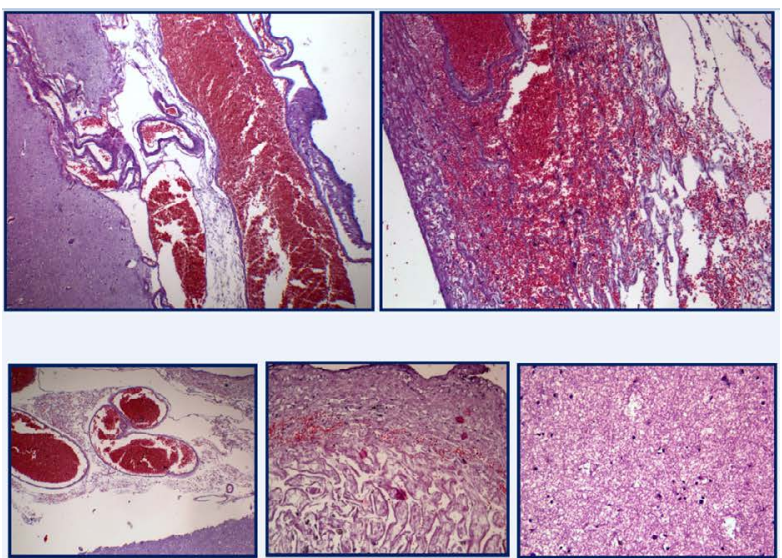

Figure 5. Dilated and congested vessels, images of angiomatosis and cortical edema. [H\&E stain].


Figure 6. Micropapillary structures with angiomatous stroma and with hyaline aspects, covered with epithelium consists of meningoendothelial cells and accasionally psammomalike body. [H\&E stain].



Figure 7. These epitelioid elements had a syncytial-like organization. The cellular elements lining the micropupils with edematous fibrous stroma and in the angiomatosis areas were characterized by finely distributed nuclear chromatin. In some cases conspicuous nucleoli with clear vacuolation and secretory vacuoles in the cytoplasm (arrow). [H\&E stain].

areas of edema and haemorrhage with hemosiderinic pigment (Figure 9a). In both lungs interstitial inflammation and marked congestion of the vessels of large, medium and small calibre were present. Aspects of hypertrophic cardiomyopathy were detected (Figure 9b).

Systematic chemical-toxicological analyses were performed on blood, urine, bile, and liver and brain samples in order to investigate the presence of any drug and/or xenobiotic that could be involved in the fatal hexitus. All biological specimens were stored at $-18^{\circ} \mathrm{C}$ until analyses. Solid tissue samples were homogenized with their own fluid after 1:1 dilution (w/v) with distilled water. The analysis of alcohol and toxic volatile or gaseous molecules was performed on peripheral blood sample by headspace-gas chromatography-flame ionization detection (HS/GC-FID); urine and blood samples were screened by immunochemical assays to highlight the presence of drugs of abuse (cocaine, opiates, cannabinoids, amphetamine/methamphetamine and derivatives, barbiturates, methadone, buprenorphine, phencyclidine, tricyclic antidepressants, fentanyl, LSD, ketamine, oxycodone, propoxyphene, methaqualone). Both HS/GC-FID and immunochemical assays resulted negative as regards screened analytes. Gas chromatography-mass spectrometric analyses aimed to highlight the presence of any other basic, neutral and/or acid chemicals in blood, urine and bile gave negative results.

\section{Discussion}

Meningioma is a extrinsic or extra- axial brain tumor that originates from meninges cells and in particular from arachnoid layer cells lying internally and externally respective of the dura mater and pia mater. Usually a benign intracranial tumor, meningioma is the one of the most common, often described in association with childhood exposure to radiation or with neurofibromatosis (2). Meningioma is usually a globular mass, with a small implant on the basis of the dura mater. The latter can be wider, especially after long time since from the development of the lesion, as well as the degree of vascularization

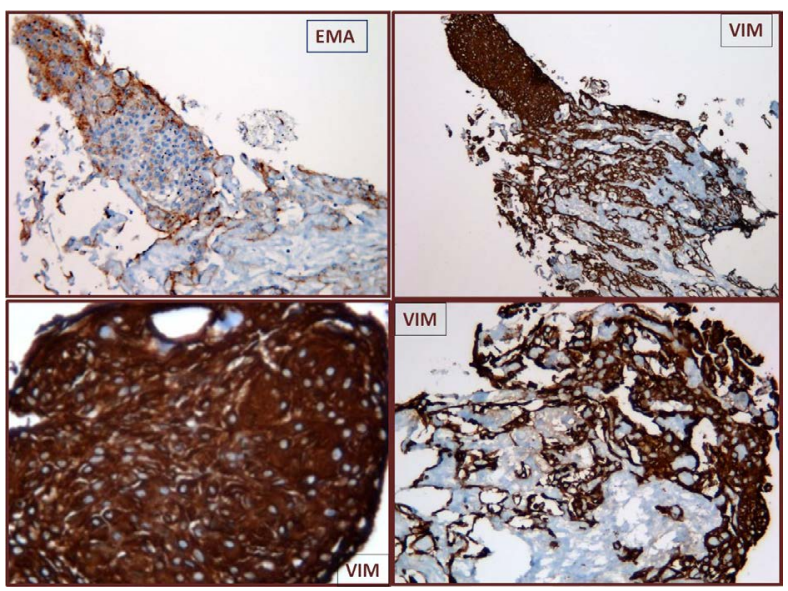

Figure 8. Immunohistochemistry showed a diffuse positivity for anti-vimentin (VIM) and focal for anti-EMA.
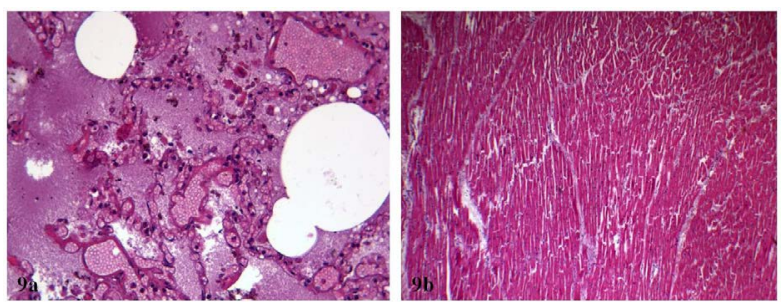

Figure 9a and 9b. (a) pulmonary diffuse areas of edema and haemorrhage with hemosiderinic pigment; (b) aspects of hypertrophic cardiomyopathy . [H\&E stain]. 
can be variable and therefore can explain the bleeding. The majority of meningiomas occurs close to the bones of the skull, causing long-term bone hyperproliferation or an invasion of bone by tumoral cells [2]. Its intracranial growth is characterized by a slow expansion that results in clinical symptoms or signs of early onset. In fact, signs of intracranial hypertension, epilepsy (especially in adult males) and focal neurologic simptoms may arise some months or even years before diagnosis. The most common sites are: the falx, convexity, the wing of the sphenoid , the olfactory shower, supra saddle, the clivale, the forame magnum, the intraventricular and spinal and posterior cranial fossa, and the parasagittal, as in the case described in the present paper.

When diagnosed by CT or MRI methods, the presence of hydrocephalus and perilesional edema are evidenced. Edema is diffusely found on histologic examination.

The perilesional edema is a consequence of vascular endothelial growth factor (EGF) secreted by the tumor cells themselves, that is responsible for the characteristic "angiomatous" appearance associated to the lesion, as in the case described here.

Generally, meningioma is considered biologically as a spaceoccupying lesion and its symptomatology is characterized by symptoms from mass effect. Conversely, in the present case the patient presented small and multiple meningiomas without symptoms of mass effect.

Morphological characterization through the light microscopy analysis differentiates between the following histological types: syncytial, fibrous, psammomatos, melanocitic, angioplasty, including the form "meningothelial meningioma" $[2,3]$. The terminology multiple intracranial meningiomas should be used only when two or more meningiomas occur either simultaneously or sequentially in different locations [4,5]. With the introduction of CT the incidence has increased but with the advent of MRI, the incidences are reported to be even higher because MRI is more helpful than CT in detecting the tumors, particularly the ones located in posterior fossa, skull base, and higher vertex area, especially when they are small. From 1977 to 2013 only 23 cases were described in a particular series of patients [6]. One of the relevant etiological factor that is important in the development of meningiomas is genetics. Studies have reported that the deletion of the chromosome 22 in patients with type 2 neurofibromatosis and in up to $50 \%$ of solitary meningiomas is connected with the appearance of multiple meningiomas [7]. The second etiological factor is represented by hormones. A number of papers show a higher frequency rate of meningiomas in women. An associated factor is the action of progesterone on progesterone receptors found in $80 \%$ of meningiomas, leading to an increase during the luteal phase of the menstrual cycle and during pregnancy [8]. In our case, the patient was male with no evidence of neurofibromatosis and behavioral disorders, and the diagnosis was formulated after his death. Autopsy examination showed varied location of the lesions, supratentorial convexity and the parasagittal falx particularly and millimeter-sized. Histologically, multiple meningiomas do not differ from the solitary types [9]. The most common histological types reported in multiple meningiomas include psammomatous, fibroblastic, meningothelial, and transitional types [10]. In our case, the histological type was a meningothelial meningioma.

Asymptomatic meningiomas are described in the literature and the annual absolute growth rate ranges from 0.73 to $1.67 \mathrm{~cm}$ per year and the tumour doubling time ranges from 1.19 to 6.81 years. This growth varies with age, is minimal, and may thus be observed without surgical intervention unless specific symptoms develop [1]. Frequently the symptoms are caused by cerebral edema that is accompanied.

\section{Conclusion}

In the here reported case, the nonspecific symptoms depended on a micropapillary form and widespread and not on a single space-occupying lesion such as is generally the case. Intracranial hypertension, epilepsy and behavioral disorder were underestimated in the socio-culturaly context for the cause of death. The histological diagnosis of multiple meningioma meningoteliale was in agreement with the literature.

\section{References}

1. Nakamura M, Roser F, Michel J, Jacobs C, Samii M (2003) The natural history of incidental Meningiomas. Neurosurg 53: 62-70. [Crossref]

2. Burger Peter C, Scheithauer Brand W. Tumors of the central nervous system. (AFIP atlas of tumor pathology) 7 . Series IV.

3. Russel DS, Rubinstein LJ (1977) Pathology of Tumors of the Nervous System, Williams and Wilkins, Baltimore, Md, USA.

4. Boylan SE, McCunniff AJ (1988) Recurrent meningioma. Cancer 61: 1447-1452. [Crossref]

5. Turgut M, Palaoglus S, Ösmam OE, Gurçay G (1997) Multiples Meningiomas of the central nervous system without the stigmata of neurofibromatosis: clinical and therapeutic study. Neurosurg Rev 20: 117-123. [Crossref]

6. Koech F, Orege J, Ndiangui F, Macharia B, Mbaruku N (2013) Multiple intracranial meningiomas: a review of the literature and a case report. Case Rep Surg 2013: 131962. [Crossref]

7. Black P, Morokoff A, Zauberman J, Claus E, Carroll R (2007) Meningiomas: science and surgery. Clin Neurosurg 54: 91-99. [Crossref]

8. Gruber T, Dare AO, Balos LL, Lele S, Fenstermaker RA (2004) Multiple Meningiomas arising during long-term therapy with the progesterone agonist megestrol acetate. Case report. J Neurosurg 100: 328-331. [Crossref]

9. Domenicucci M, Santoro A, D'Osvaldo DH, Delfini R, Cantore GP, et al. (1989) Multiple intracranial meningiomas. J Neurosurg 70: 41-44. [Crossref]

10. Eljamel MS, Foy PM (1989) Multiple meningiomas and their relation to neurofibromatosis. Review of the literature and report of seven cases. Surg Neurol 32: 131-136. [Crossref]

Copyright: (C2016 Gelsomina M. This is an open-access article distributed under the terms of the Creative Commons Attribution License, which permits unrestricted use, distribution, and reproduction in any medium, provided the original author and source are credited. 